\title{
Scientific Computing
}
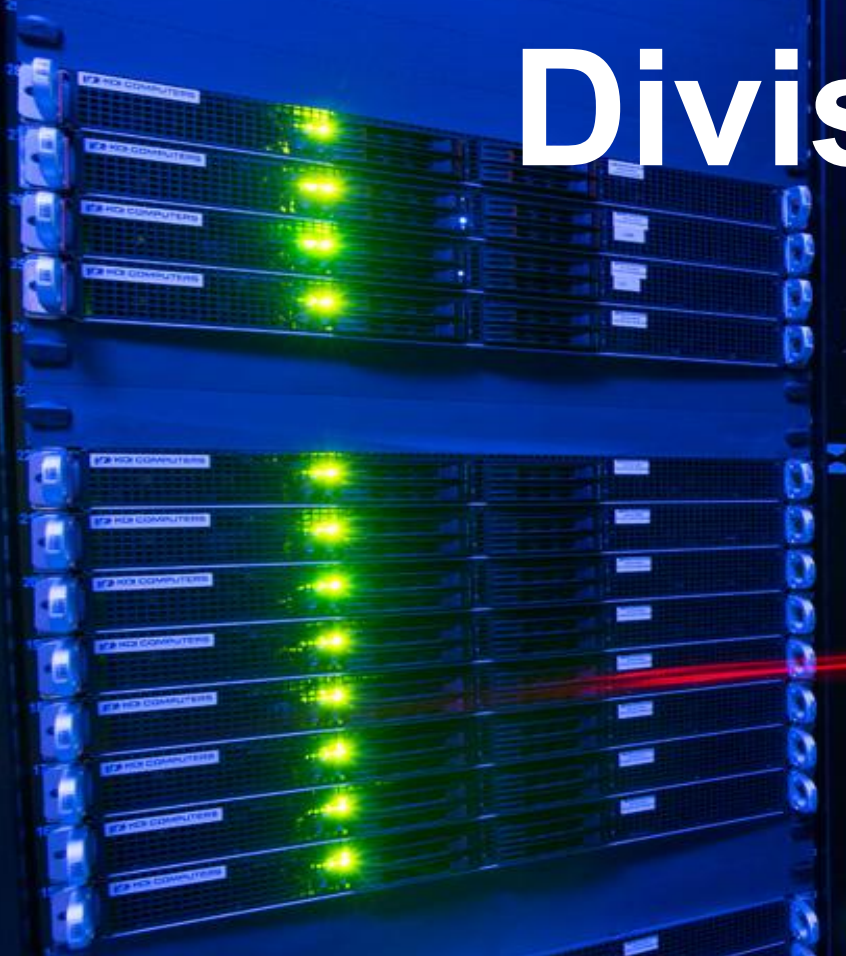

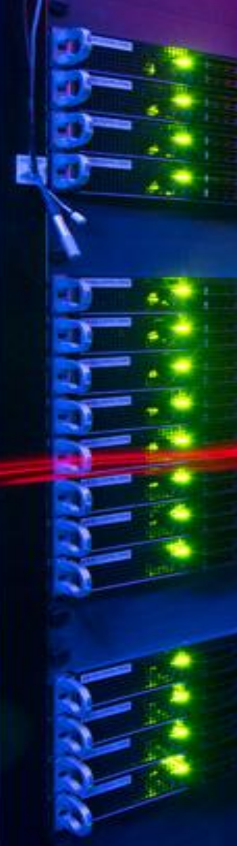

New Perspectives 2021 aleksand@fnal.gov 


\section{Talk Outline}

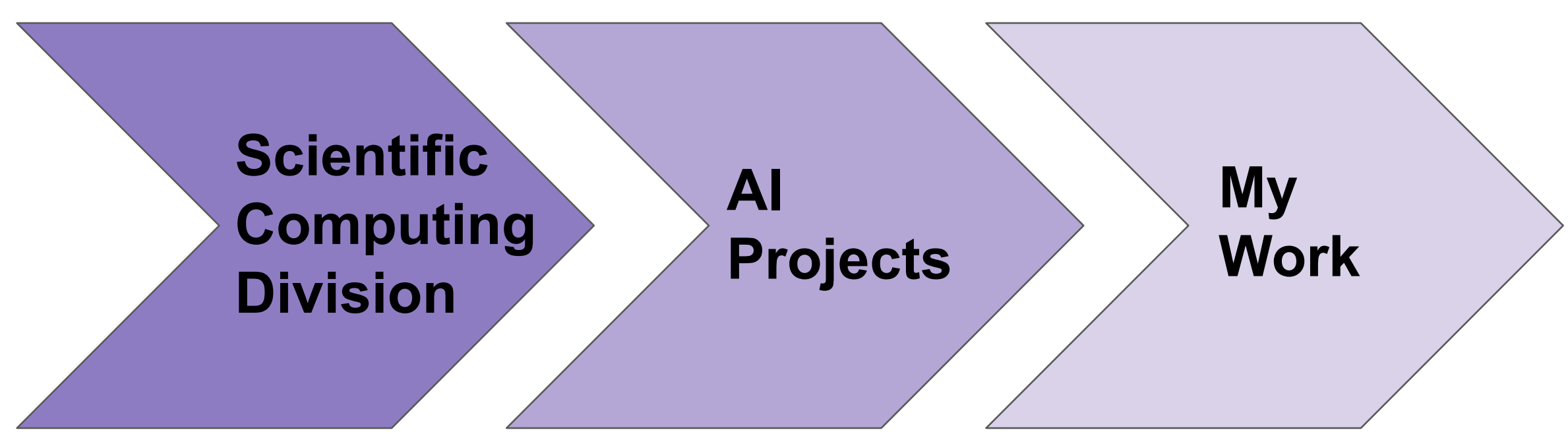



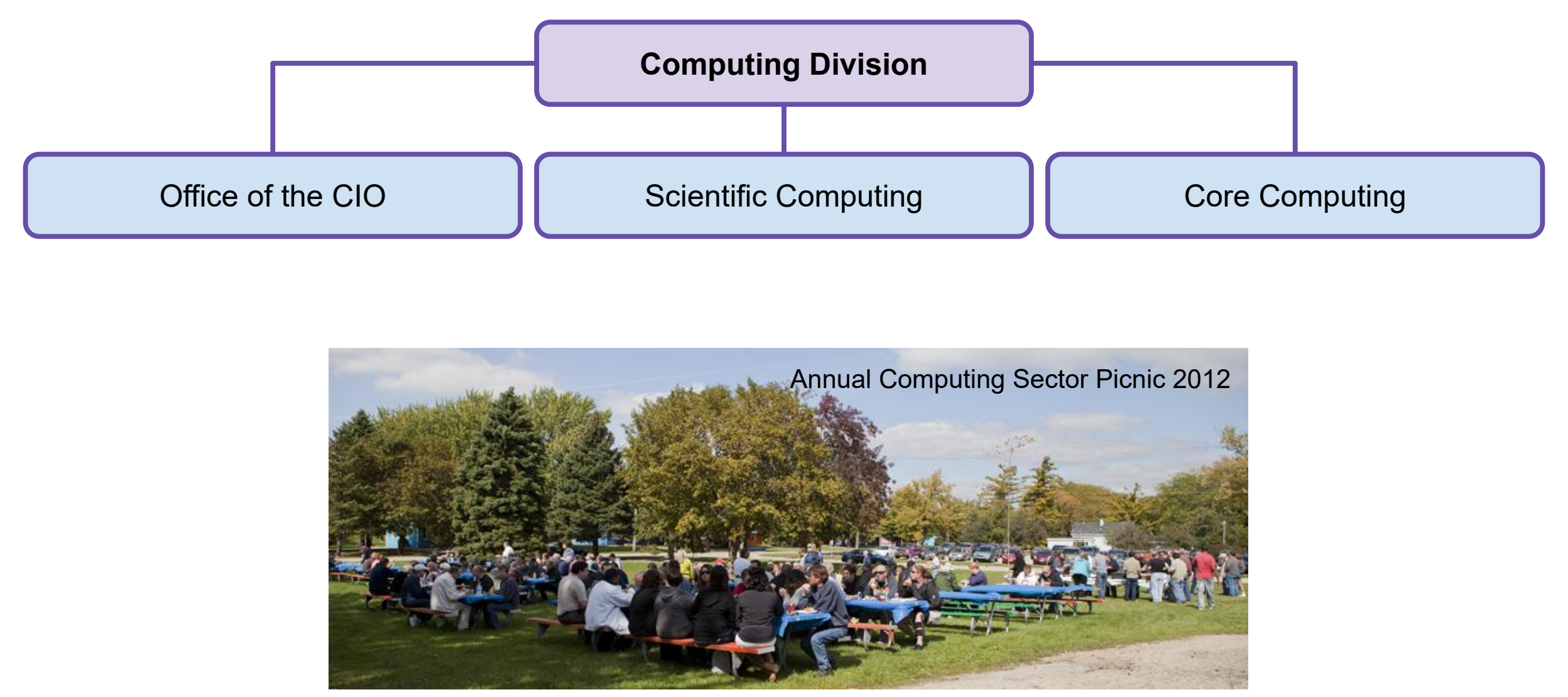


\section{Al capabilities and focus areas}

Uncertainty quantification

Learning on graphs
Coprocessors for fast inference Distributed training

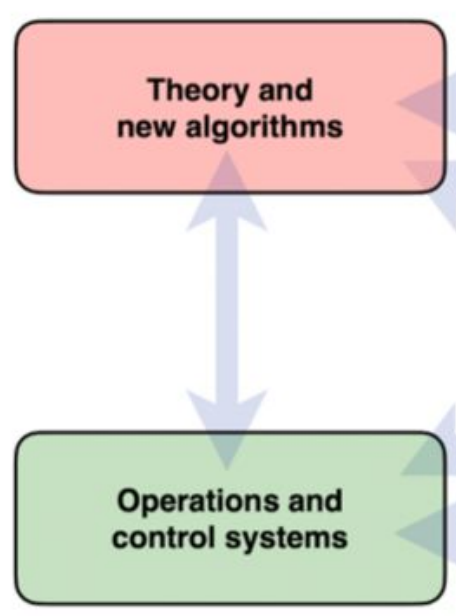

Self operating telescopes

Experiment controls

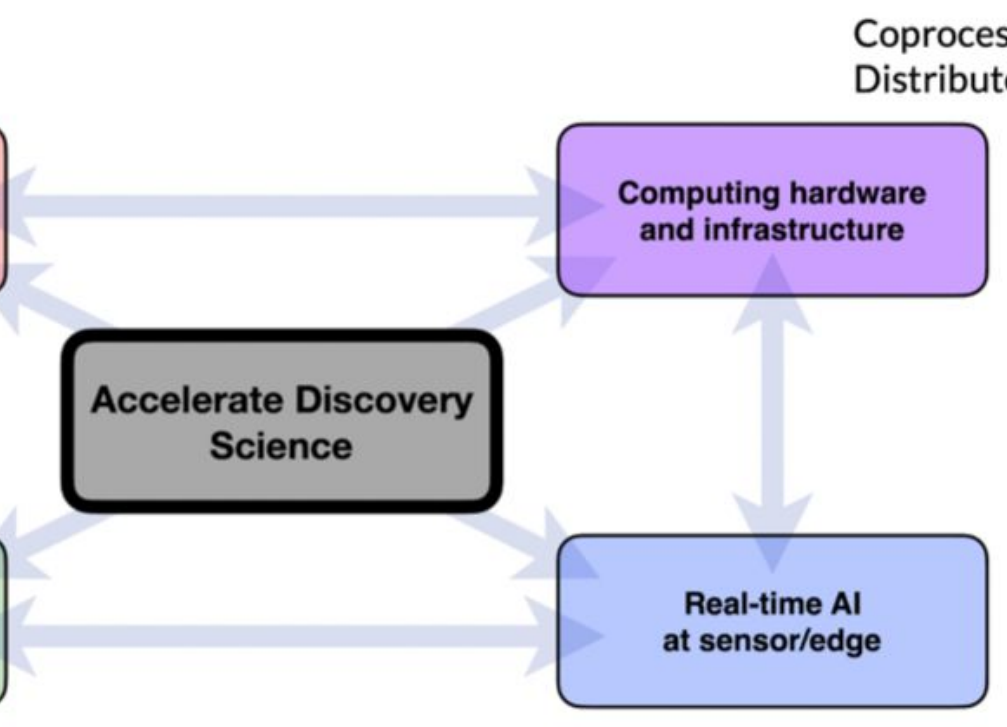

hls $4 \mathrm{ml}$ and FPGAs

ASIC codesign 


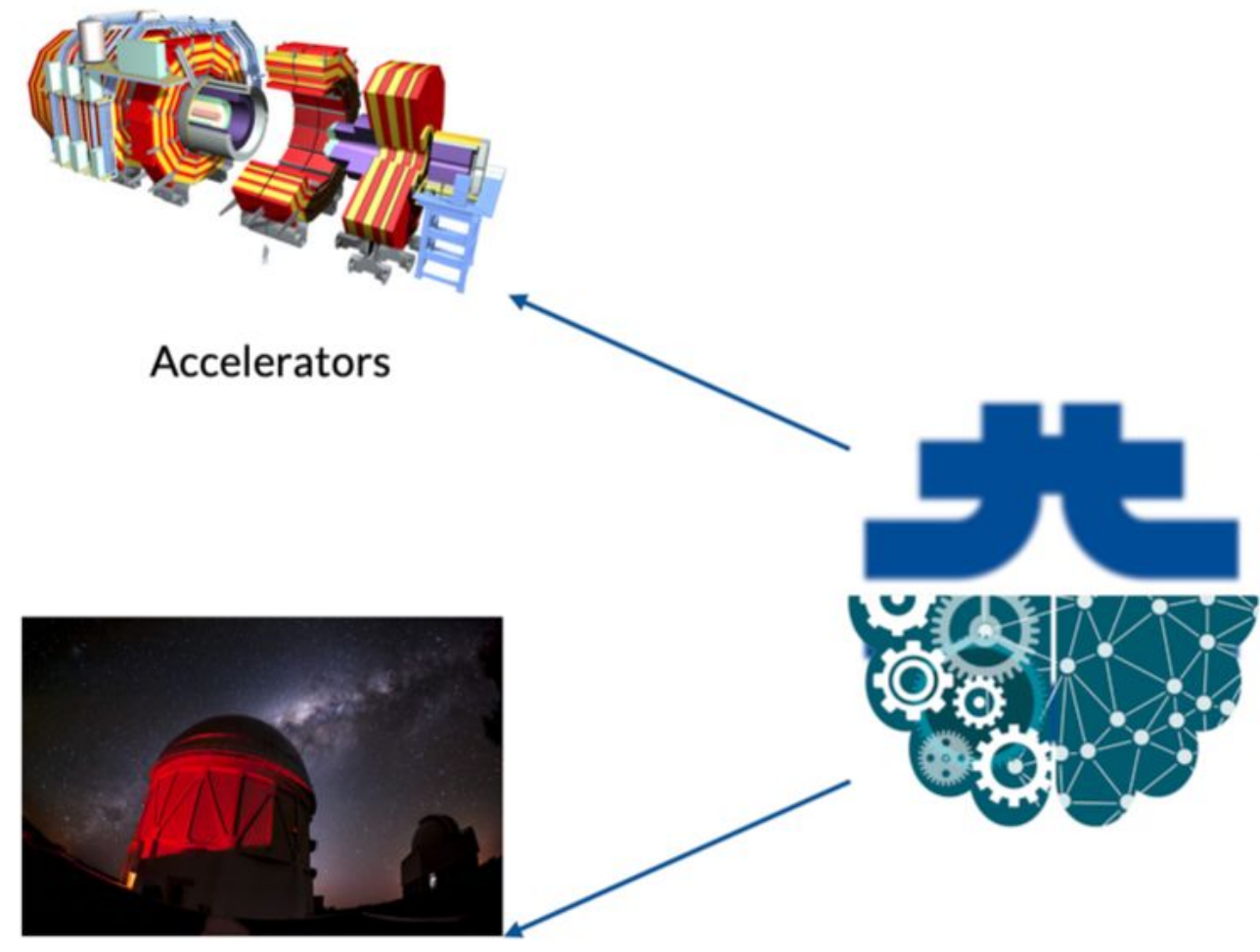

Astronomy

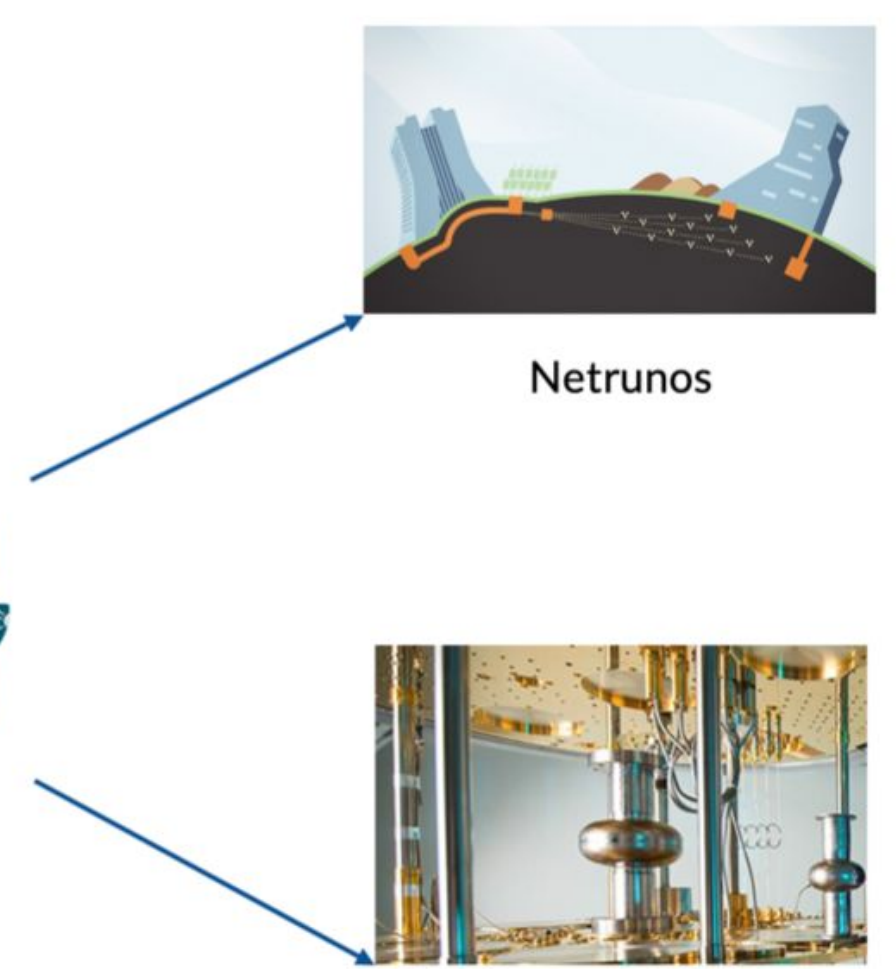

Quantum Science 


\section{My science interests}

- Formation and evolution of structure in the Universe

- Build robust, trustworthy and understandable Al

- Leverage and use all available datasets - learn from their similarities and differences

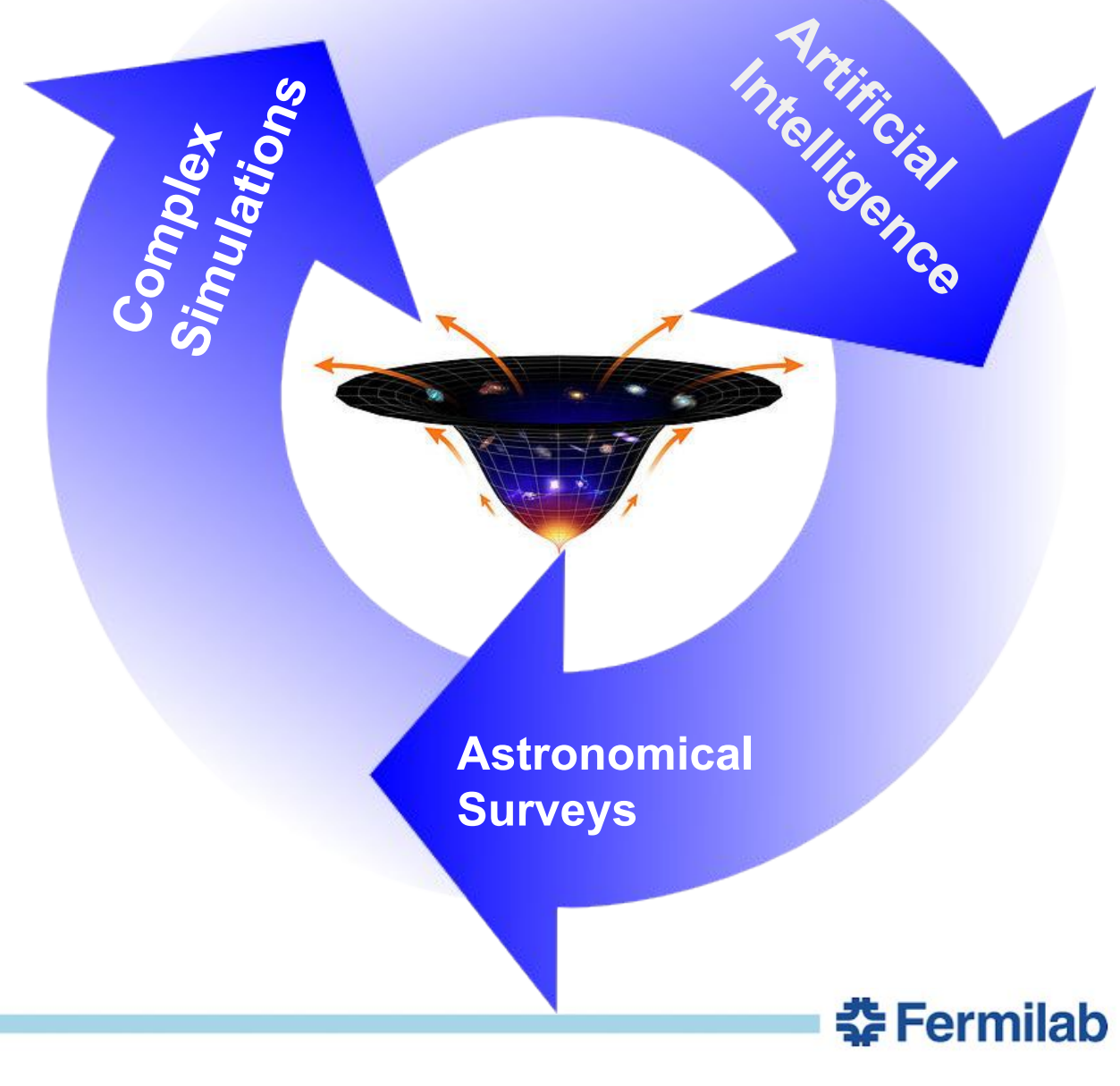




\section{My Projects}

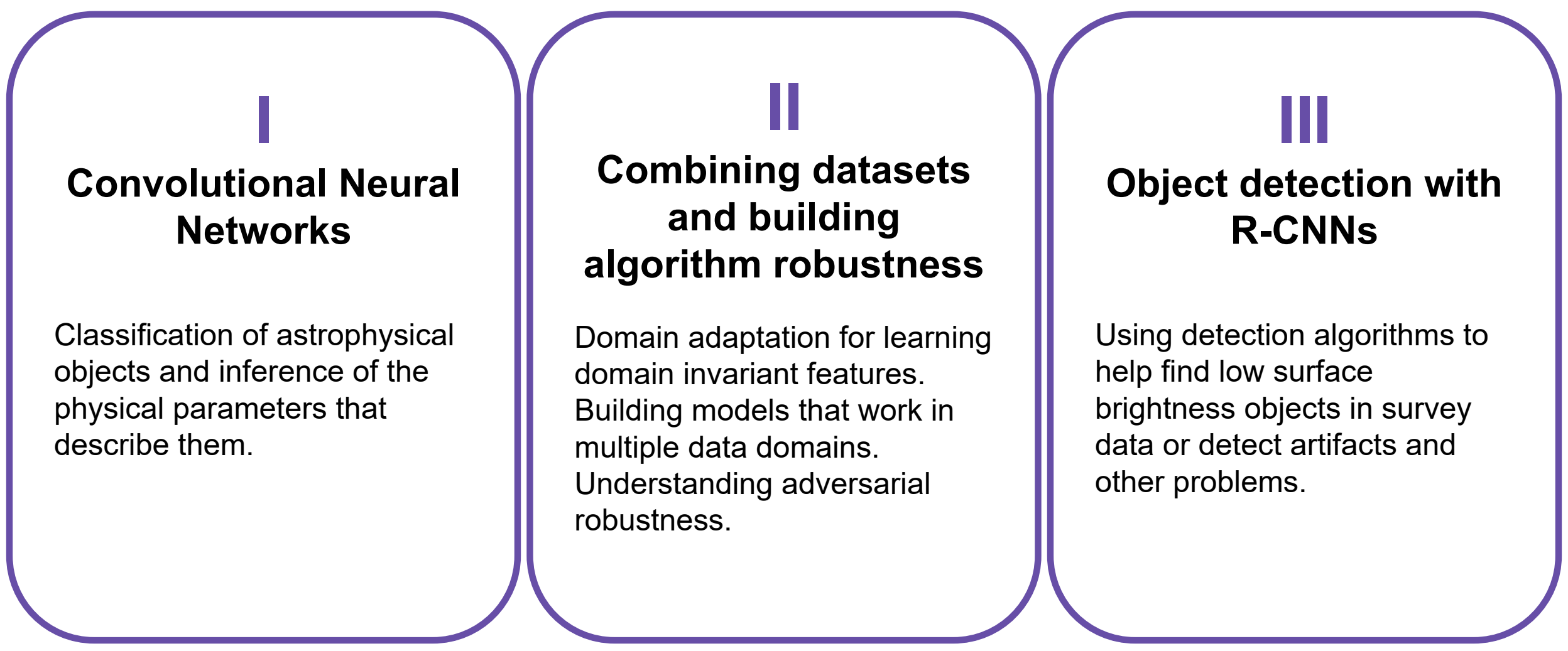




\section{My Projects}

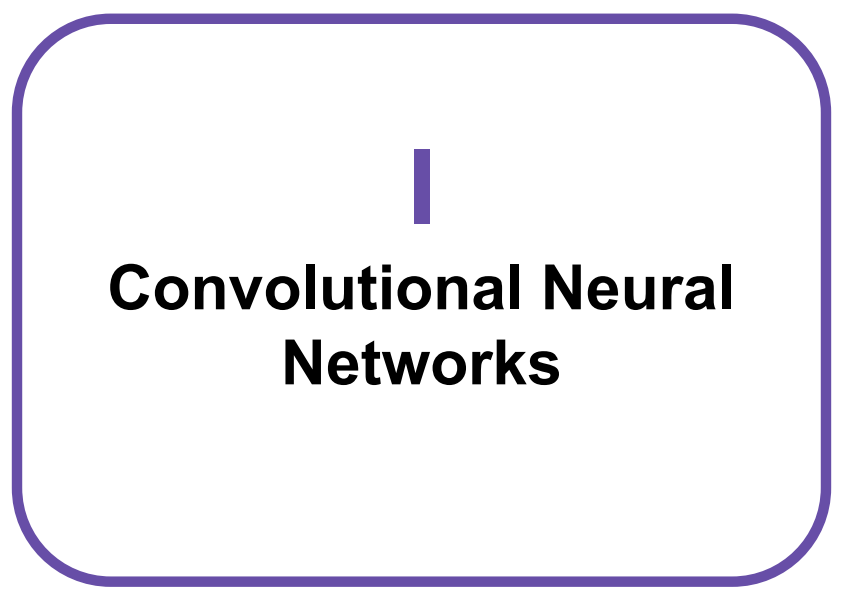

I "DeepMerge": Ćiprijanović et al. 2020

"DeepShadows": Tanoglidis et al. 2020.
- Using deep learning (CNNs) to distinguish between merging and non-merging galaxies, which are crucial for understanding of galaxy evolution.

- We show it is possible even for very distant galaxies!

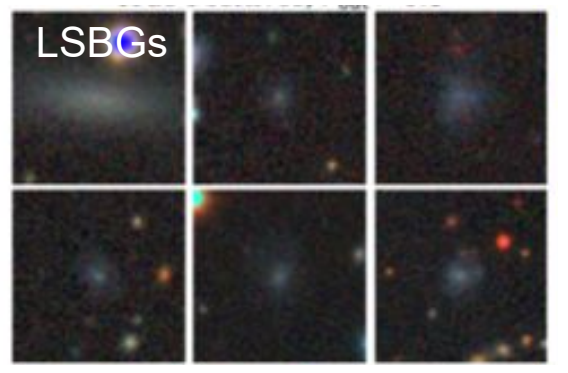

- Using deep learning to distinguish

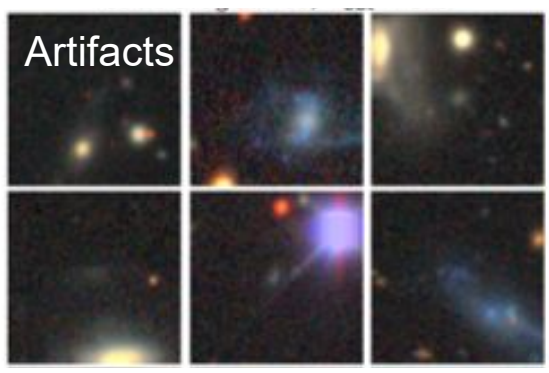
low surface brightness galaxies (LSBGs) from artifacts in DES data.

- Crucial for building their catalogues, LSBGs might be the most numerous type of galaxies!

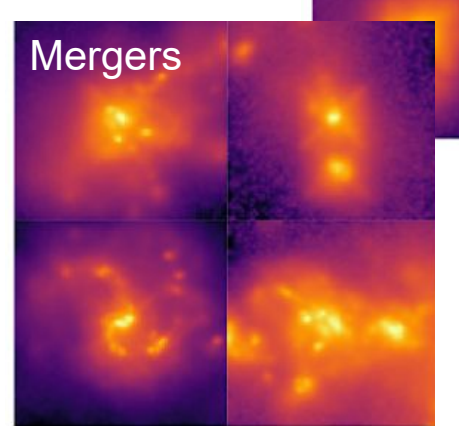




\section{My Projects}

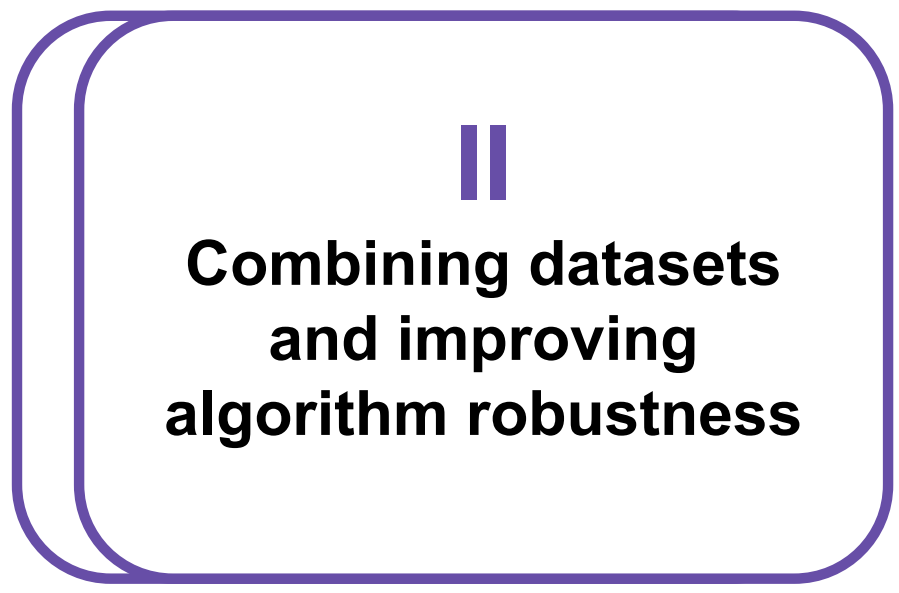

You heard more in from Kathryn Downey the previous talk!

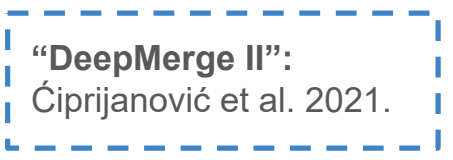

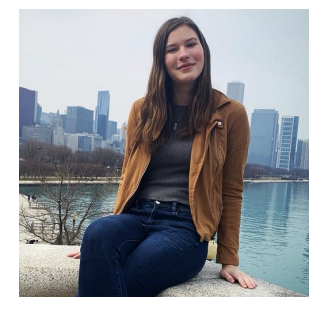
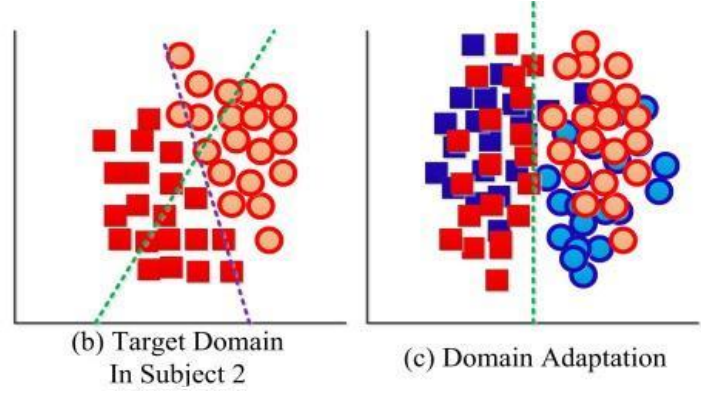

Source Domain In Subject 1

- Domain adaptation methods allow us align data distributions and find common decision boundary.

- We can use combine knowledge from simulation with new and unlabeled observations from astronomical surveys!

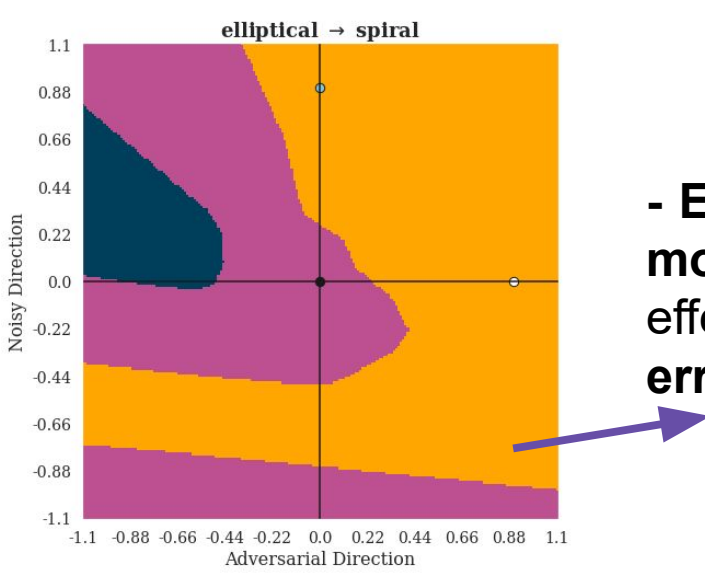




\section{Big thanks to all my amazing collaborators!}

Fermilab
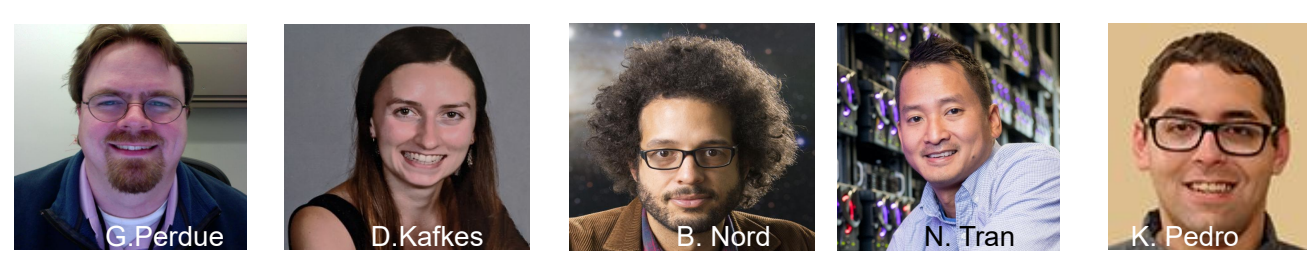

University of Chicago
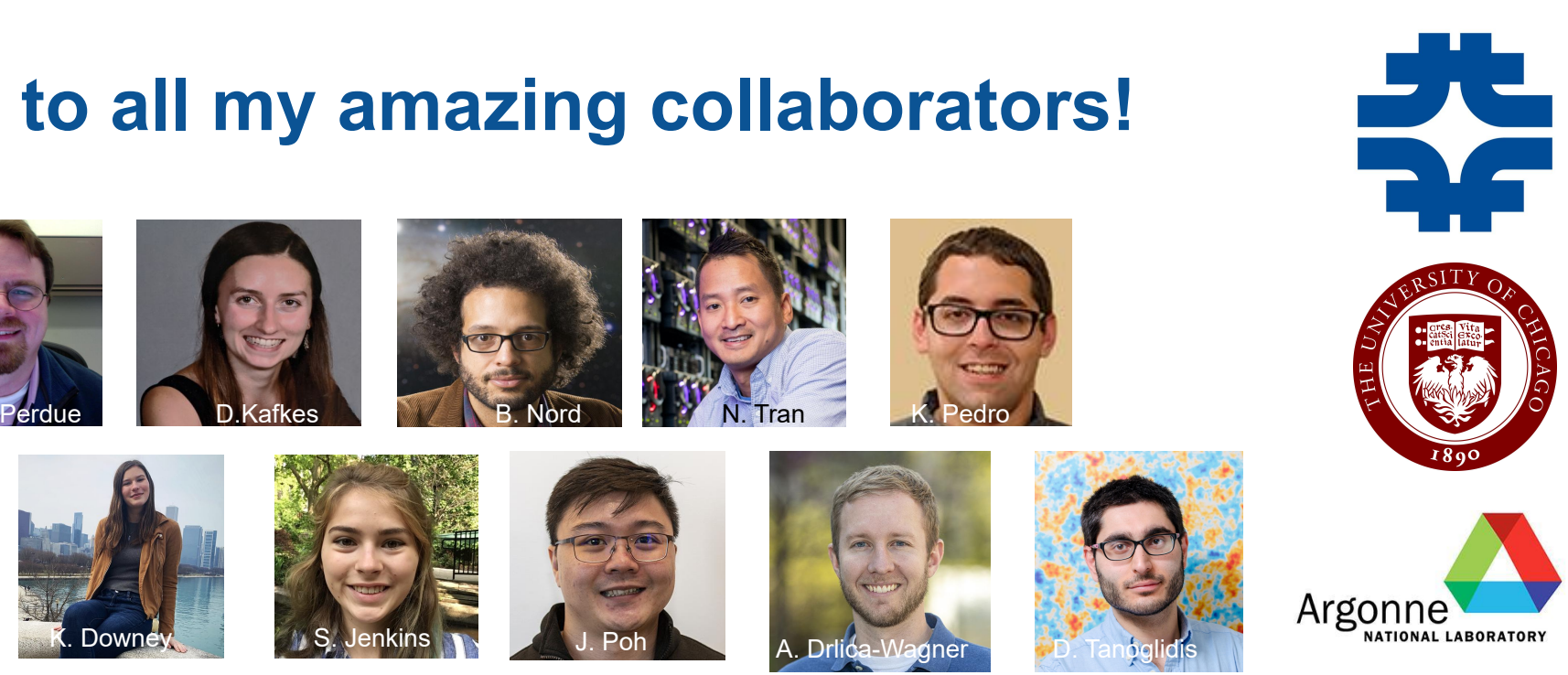

Argonne, Oakridge
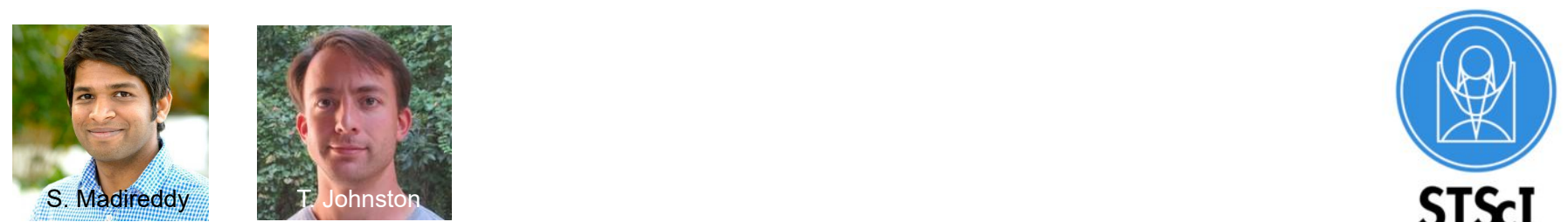

STSCI

Space Telescope Science Institute
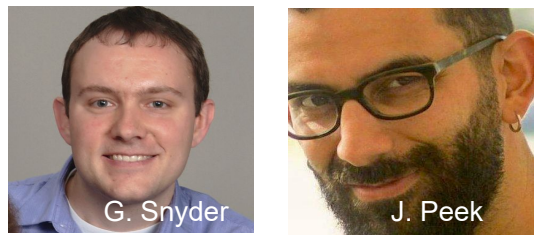

"OAK

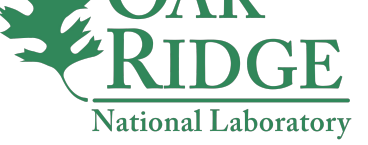





\section{Thank you!}

aleksand@fnal.gov 


\section{Al Initiative}

\section{Scientific Computing Division}

Cross-cutting projects and initiatives

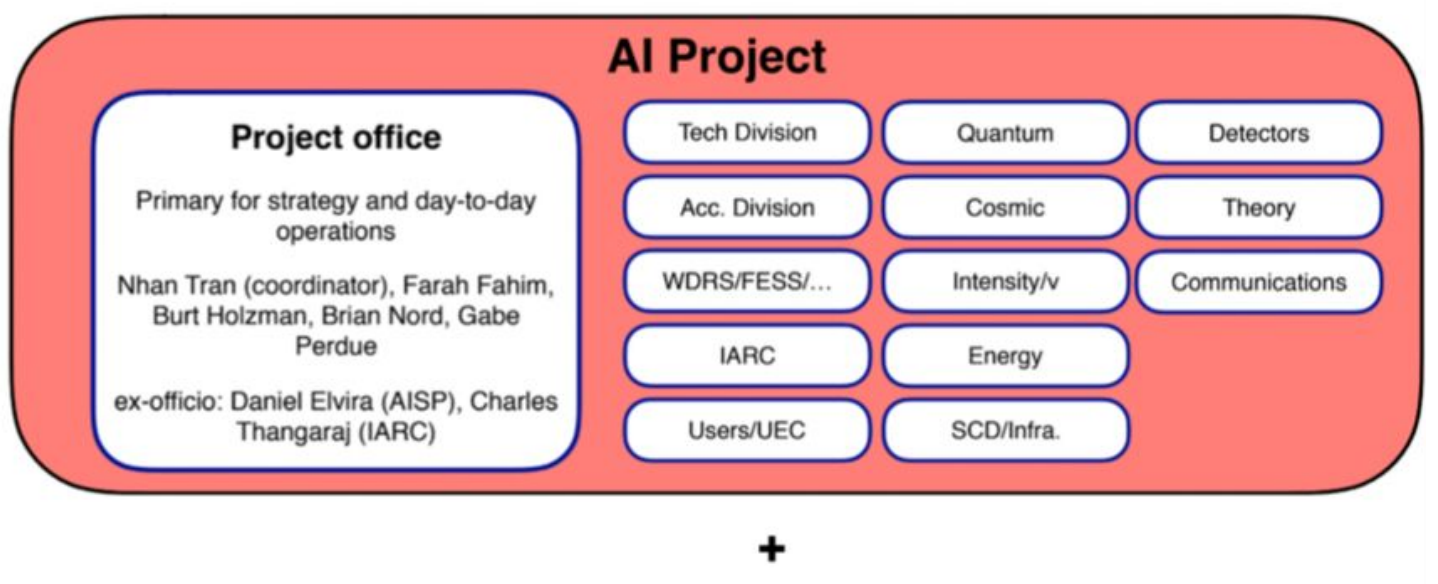

Liaisons as links across the laboratory

Formal home in SCD but engaging the entire laboratory. 